\title{
User command acquisition based iot automatic control system
}

\author{
Inshik Kang1, Hyunok Song ${ }^{2}$, Hoekyung Jung ${ }^{3}$ \\ ${ }^{1}$ Department of Cinematography, Korea University of Media arts, Sejong, South Korea \\ ${ }^{2}$ Dasomsoft, 1906-ho, 28 Daehak-ro, Yousung-gu, Daejeon, South Korea \\ ${ }^{3}$ Department of Computer Engineering, Paichai University, Daejeon, South Korea
}

\begin{tabular}{l}
\hline Article Info \\
\hline Article history: \\
Received Aug 9, 2018 \\
Revised Oct 31, 2018 \\
Accepted Nov 19, 2018 \\
\hline Keywords: \\
Control Command \\
Heterogeneous Device \\
IoT \\
Sensor Data \\
Threshold
\end{tabular}

\begin{abstract}
Recently, researches are being conducted to build a smart environment using various sensors and devices and to provide customized services to users through IoT (Internet of Things). The existing IoT system operates the device only by using the threshold value. So, there are problems that device operates when a service that does not consider a user characteristic is provided or when user not necessary. In this paper, to solve these problems, the system collects the remote control commands and compares it with the value of device operation threshold and sets the changed threshold value. In addition, devices not registered in the server constitute the environment by linking with existing task. Therefore, it is possible to provide a customized service to the users.
\end{abstract} All rights reserved.

\section{Corresponding Author:}

Hoekyung Jung,

Department of Computer Engineering,

Paichai University,

Daejeon, South Korea.

Email: hkjung@pcu.ac.kr

\section{INTRODUCTION}

Today, IoT is evolving into a system for intelligent services where people, processes, data and objects are interconnected. In the past, it only supported inter-object communication via M2M (Machine To Machine), but it is evolving as a step to decide between the objects. It is also a step of recognizing and controlling the situation in the surroundings where the person is not aware $[1,2,3,4]$. To provide these services, it is necessary to use various sensors to measure environmental data. In addition, the data generated by objects and people included in the IoT network is increasing exponentially, and there are requirements to be solved by IoT systems such as real-time processing capability and accurate semantic information [3,5,6,7].

Existing IoT systems are transmit data measured through a sensor to a server, load it in a database, and extract and provide data according to the user's request. Such a system operates the device through a threshold value in the server, but does not consider the characteristics of the user, and thus the device operates even when the user does not want it [8-12].

In order to solve this problem, in this paper, when the user remotely controls the device, the command is collected and the existing threshold value and analysis are performed to set the threshold value suitable for the user. Also, threshold are loaded into the database and provided to the user application. In addition, heterogeneous devices transmit sensor data and device information to the server and are linked to existing tasks when automatic control and remote control occur. As a result, unnecessary task can be reduced and a customized service can be provided to users. 


\section{SYSTEM DESIGN}

This chapter covers system design. The system is an automatic control system and is a system in which the threshold changes when a user operates the device through remote control. Arduino is equipped with sensors and devices and communicates with the server via Wi-Fi. The server compares the sensor data with the threshold value to determine whether a task has occurred or not, and transmits a control command to the device. And the server loads the results into the database and provides them to the user application. Figure 1 shows the system architecture.

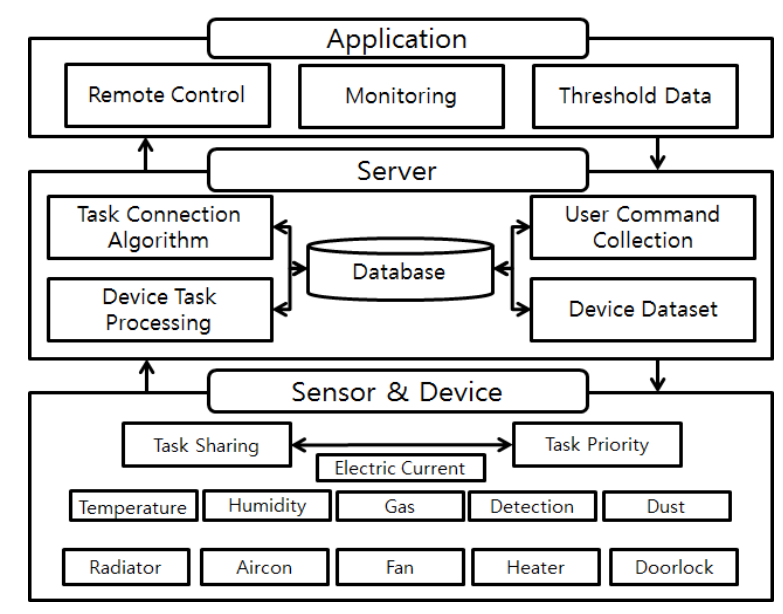

Figure 1. System architecture

The sensor and the device measure the surrounding environment data in the home and transmit it to the server. When the environment change, the device operates by the threshold value and keeps it in a pleasant state. The server consists of a database and modules for data processing. The database stores sensor data for monitoring, device status, and operation thresholds. The application can monitor and control the sensor data and device status by the user. You can also check device-specific thresholds and power usage. Figure 2 shows a flow diagram of the algorithm for associating heterogeneous devices with existing tasks.

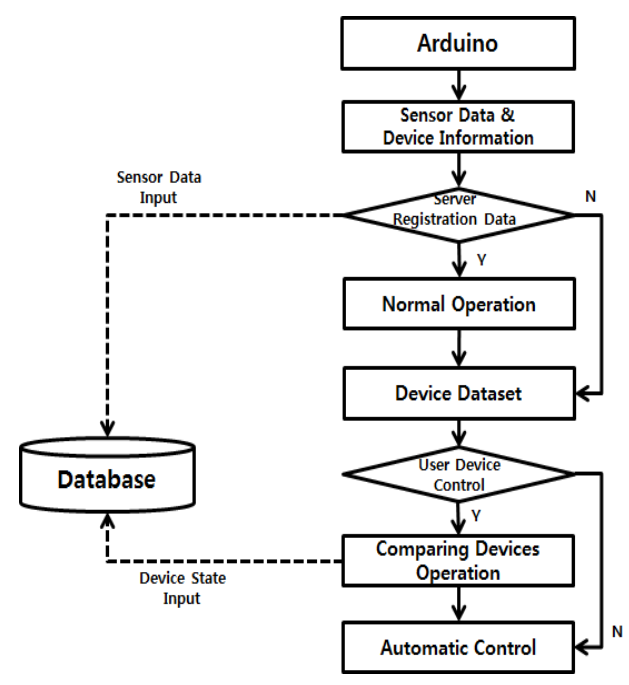

Figure 2. Device connection algorithm flowchart

Data about sensor and device connected to Arduino is transmitted to server. The server judges whether or not the corresponding data is registered in the server. If it is registered, it does not need to associate with the existing task and the normal operation proceeds. If not registered, a dataset for the device 
is created and loaded into the database. Confirm that the added heterogeneous device is operating. When an existing operation is in operation, it is compared whether the device changes environment analyze the operation of the device. If the task is not running, it operates as an automatic control task. Therefore, the user advances the connected each time the remote control of heterogeneous devices. Figure 3 shows the task processing priority in the server.

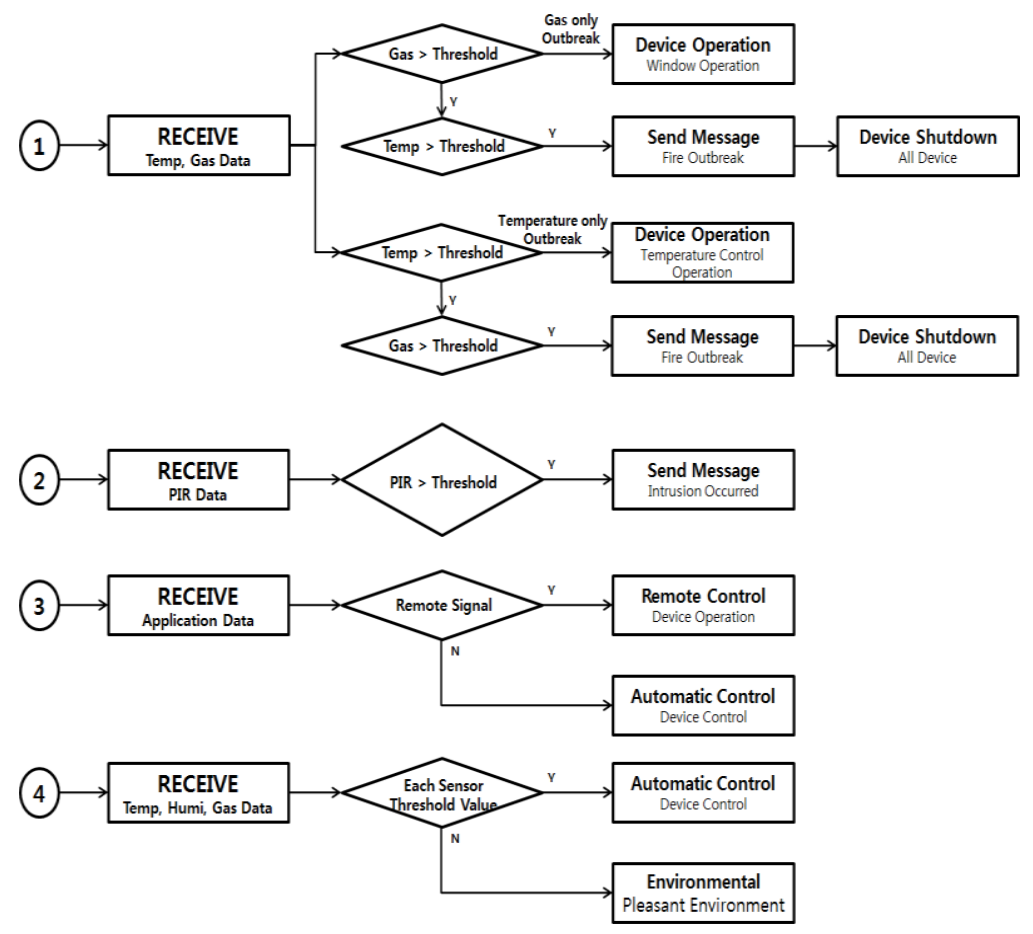

Figure 3. Step-by-step task priority setting

The first priority task is fire caused by temperature or gas sensor. If the temperature data does not exceed the threshold value and the gas threshold value is exceeded, it sends a message to the user that the gas has leaked and operates the window device to ventilate. If the threshold of the temperature is exceeded and the threshold of the gas is exceeded, it is identified as a fire, and after the message of fire occurrence is sent to the manager, all devices stop. If the temperature only exceeds the threshold, the device for temperature control operates.

The second priority task is a door lock control by human detection sensor. If there is a user inside the home, the device for temperature control or ventilation in the server will operate. If there is no user in the home, the device does not operate. In the case of a fire, it is an operation that is identified even if the user is not inside home.

The third priority task is remote control using user application. When a device remote control signal is generated by a user, a control command is transmitted to the corresponding device, and otherwise, the device operates by automatic control. Also, if the device is operated due to remote control, the device with lower priority stops.

The fourth priority task is an automatic control using temperature, humidity and gas sensor. The sensor data is used to determine whether an operation has occurred through the threshold value in the server, and a device for environmental control operates when temperature regulation and ventilation work occurs.

\section{SYSTEM IMPLEMENTATION}

This chapter covers the implementation of the proposed IoT system. The application of the proposed system is an important module for the user to monitor environment data in the home and remotely control the device. Figure 4 shows the main page and monitoring page displayed when the application is executed.

When you run the application, the initial screen will appear. Click the Monitoring button to go to the monitoring page. The monitoring page extracts sensor data and a device state loaded in the database and 
provides them to the user. The device is remotely controlled by the ON and OFF buttons. When the user presses $\mathrm{ON}$, the state of the database is updated, and the command is collected for the threshold change and the command is transmitted to the device. Figure 5 shows the threshold page converted by the user's remote control.

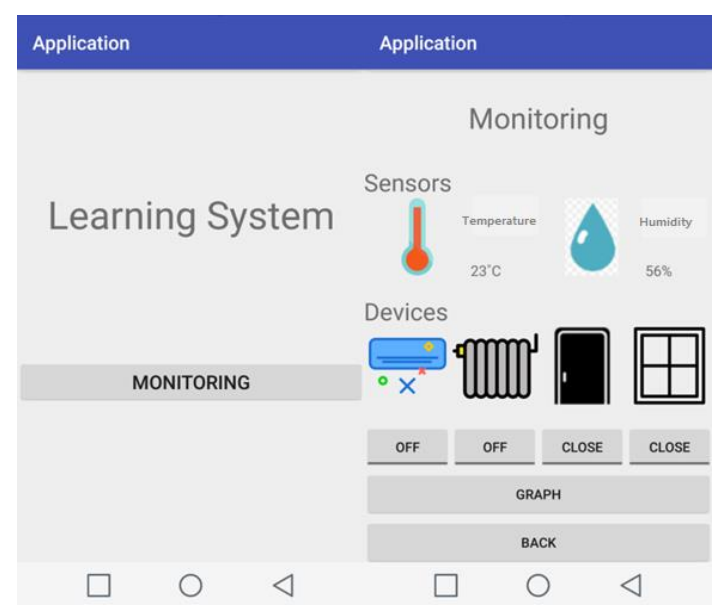

Figure 4. Main page and monitoring page

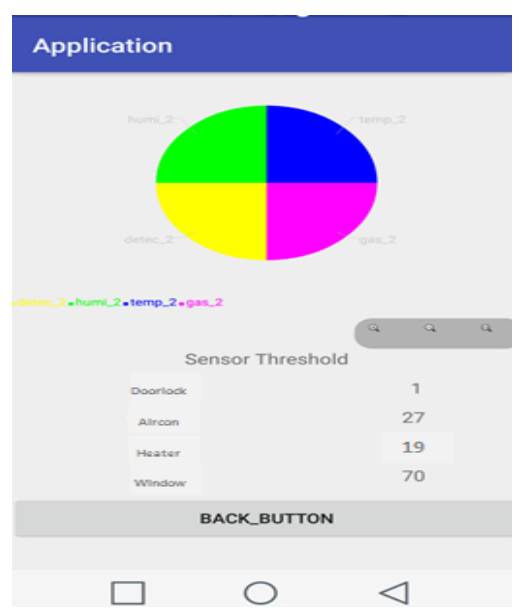

Figure 5. Remote control threshold page

It extracts and provides device state data and threshold values stored in the database. This allows the user to see the changing threshold as the device operates. Figure 6 shows the overall system prototype.

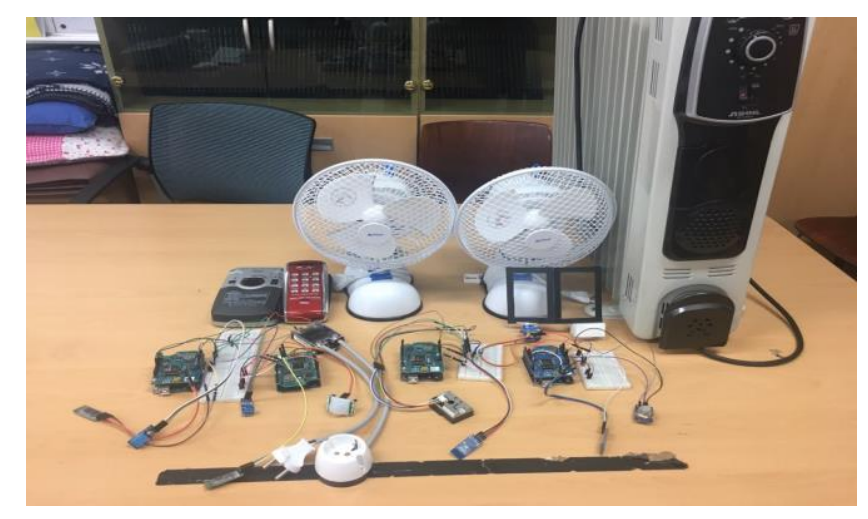

Figure 6. Prototype of the proposed system

The prototype of the proposed system is composed of four kinds of sensors: temperature sensor, humidity sensor, gas sensor and human detection sensor. The devices that operate are air conditioners, heaters, windows and door locks. The data measured through the sensor is initiated through the thresholds in the device and the system proceeds through sharing and comparison. The temperature and humidity sensor operate the fan and the heater related to the temperature control, and the gas sensor performs the ventilation according to the gas generation. The human detection sensor confirms whether there is a user in the management place and controls the door lock.

\section{REVIEW}

Existing systems have been designed to utilize various sensors to configure a smart environment and provide automation services. However, there is a problem that it operates with a fixed threshold value without considering a command or characteristic of a user. In addition, when a heterogeneous device is added and associated with existing work, there is a disadvantage that the server must be reconfigured. 
In this paper, in order to solve the problems of the system which does not take into account the characteristics of the user in the IoT environment, this study collects the sensor data at the time of command when the control command of the user occurs. The threshold value to be operated by the corresponding device is analyzed and the threshold value is converted. In addition, it collects sensor data and device information of devices not registered in the server, and works with existing registered devices. Table 1 shows the dataset for the experiment.

Table 1. Experimental dataset

\begin{tabular}{cc}
\hline System Type & Description \\
\hline Existing System & Temperature Threshold $=19^{\circ} \mathrm{C}$ below \\
& Device $=$ Heater \\
& (Auto Control \& Remote Control) \\
Data Processing $=$ Server \\
Throphold $=19^{\circ} \mathrm{C}$ below \\
Device $=$ Heater \\
(Auto Control \& Remote Control) \\
Data Processing = Server
\end{tabular}

It is an experiment in which the operating threshold of the heater device is changed due to the user's remote control by configuring the existing system and the proposed system in the same environment. Figure 7 shows the graph of the threshold change according to the remote control of the existing system. Figure 8 shows the graph of the proposed system.

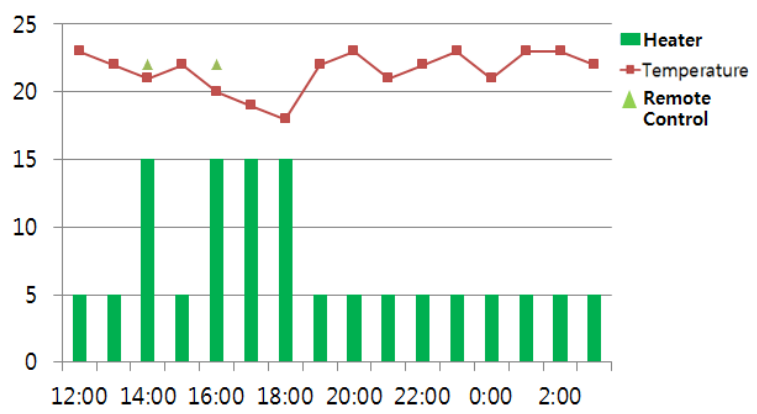

Figure 7. Graph of existing system threshold changes

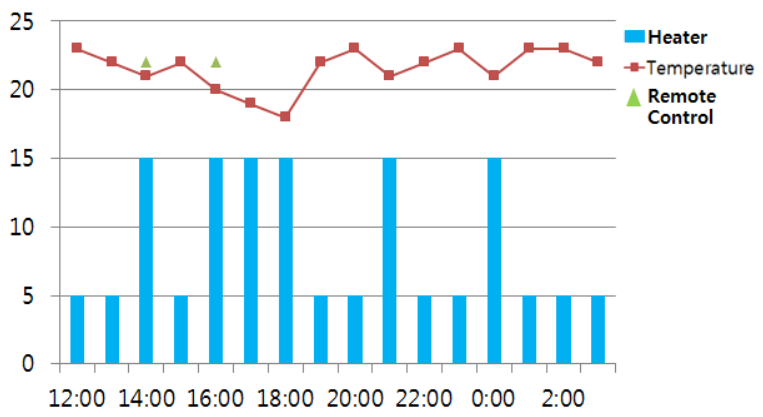

Figure 8. Graph of proposed system threshold changes

An experiment using a dataset is an experiment in which the user proceeds to remote control at a threshold value at which the heater does not operate and checks whether the threshold value has changed. In the existing system, the heater device was operated when the time was $14: 00$ and the temperature was $21^{\circ} \mathrm{C}$ and the time was $16: 00$ and $20^{\circ} \mathrm{C}$, and it was confirmed that there was no change in the threshold value. The proposed system also operated the heater device remote control at 21:00 and $20^{\circ} \mathrm{C}$ at 14:00 and 16:00 to measure the threshold change. In the proposed system, the threshold value of the heater which was operated only under the conventional $20^{\circ} \mathrm{C}$ was changed, and the heater was operated at 21:00 and 00:00. 
Through the experiment, an operation is performed based on the remote control command of the user, and the customized service can be provided by changing the fixed threshold value to the user customized threshold value.

\section{CONCLUSION}

In the existing system, devices in the home are connected to a single network, and control commands are transmitted through the server. Such a system has a drawback in that it provides a service that does not take into consideration the characteristics of a user because the device operates through a threshold value fixed in the server. Also, when a new device is configured in a new device or an IoT system utilized by a user, there is a problem that an existing server must be reconfigured or a new server must be configured.

In this paper, we proposed a system in which the threshold value of the device changes according to the user's control command. The proposed system transmits the sensor data to the server and operates the operation judgment and the device through the threshold value. When the remote control command of the user occurs, the sensor data is collected. Then, the threshold value stored in the database is extracted and analyzed. The analysis learns thresholds and proceeds by device. Accordingly, the analysis is performed every time a control command of a user is generated, and a service considering characteristics of each user can be provided. Also, when heterogeneous devices not registered in the server occur, sensor data and device information are loaded in the database. Each time the device operation proceeds, it is linked with the device operation that is in the existing operation or in the standby state. The user can monitor and control the threshold, power consumption, sensor data, and device status through the application.

We have verified that efficiency and accuracy are increased compared to existing systems. Through this, the threshold value of the device changes according to the characteristics of the user, and a new device can be learned and a service with increased convenience can be provided. Future research should be carried out by applying the proposed system to various environments.

\section{ACKNOWLEDGMENTS}

This research was supported by The Leading Human Resource Training Program of Regional Neo industry through the National Research Foundation of Korea(NRF) funded by the Ministry of Science, ICT and future Planning(No. 2016H1D5A1911091). This work was supported by the research grant of Pai Chai University in 2018.

\section{REFERENCES}

[1] W. Y. Lee, H. M. Ko, J. H. Yu, K. B. Sim. An Implementation of Smart Dormitory System Based on Internet of Things. Journal of Korean Institute of Intelligent Systems. 2016; 26(4): 295-300.

[2] M. J. Song. A Study on Business Types of IoT-based Smarthome: Based on the Theory of Platform Typology. The Journal of the Institute of Internet, Broadcasting and Communication. 2016; 16(2): 27-40.

[3] X. Bing. Key Internet of Things Technology and Application Research. TELKOMNIKA Indonesian Journal of Electrical Engineering. 2014; 12(7): 5599-5602.

[4] K. Manickavasagam. Automatic Generation Control of MultiArea Power System with Generating Rate Constraints Using Computational Intelligence Techniques. International Journal of Applied Power Engineering. 2013; 2(1): 27-38.

[5] C. Y. Hsieh. Prediction of Forest Temperature Microchange by Wireless Sensor Network Technology. International Journal of Electrical and Computer Engineering. 2012; 2(5); 632-638.

[6] S. H. Kim, M. Y. Song. Development of Academy Management Prototype System Using Mobile App. Journal of the KOREA Contents Association. 2015; 15(8): 18-23.

[7] D. W. Choi, H. J. Cho. Machine learning based motion recognition technology for intelligent IoT service. Journal of the Korea Electromagnetic Engineering Society. 2016; 27(4); 19-28.

[8] K. Cao, I. S. Kang, H. W. Choi, H. K. Jung. Reagent Cabinet Management System Using Danger Priority. Journal of Information and Communication Convergence Engineering. 2017; 12(4): 227-231.

[9] K. Cao, H.J. Lee, H.K. Jung, Task Management System According to Changes in the Situation Based on IoT, Journal of Information Processing System. 2017; 13(6):1459-1466.

[10] C. W. Son, S. B. Lee. Realization of home appliance classification system using deep learning. Journal of the Korea Institute of Information and Communication Engineering. 2017; 21(9): 1718-1724.

[11] T. H. Park, H. J. Seo, B. J. Bae, H. W. Kim. Secure Message Transmission against Remote Control System. Journal of Information and Communication Convergence engineering. 2016; 14(4); 233-239.

[12] S. L. Choi, S. J. Koh. Distributed CoAP Handover Using Distributed Mobility Agents in Internet-of-Things Networks. Journal of Information and Communication Convergence Engineering. 2017; 15(1): 37-42. 